\title{
Incidental vocabulary acquisition during reading by adult native speakers of German
}

Denisa Bordag ${ }^{1}$, Amit Kirschenbaum ${ }^{1,2}$, Andreas Opitz ${ }^{1}$ Erwin Tschirner ${ }^{1}$

${ }^{1}$ Herder-Institut, University of Leipzig, Germany

${ }^{2}$ Institut für Informatik, University of Leipzig, Germany

https://doi.org/10.36505/ExLing-2012/05/0006/000212

\begin{abstract}
In the present study, we explore the influence of syntactic complexity on incidental vocabulary acquisition. Instead of relying on introspective research methods based on self-evaluation that have been typically used previously (e.g. the Vocabulary Knowledge Scale (Paribakht \& Wesche, 1996)), we employ several psycholinguistic paradigms to investigate the acquisition of various lexical aspects of the new words (especially of phonological form and meaning) under two different acquisition conditions: a) directly within the context in which the subject first encounters the new word (self-paced reading) and b) independently from the acquisition context (lexical decision and semantic priming). The results of the present study indicate that the acquisition of new words is more successful in syntactically simple contexts.
\end{abstract}

Key words: incidental vocabulary acquisition, reading, syntactic complexity

\section{Introduction}

Incidental vocabulary acquisition, i.e., "the acquisition of vocabulary as a by-product of another activity" (Laufer, 2001), has gained considerable attention in past decades, especially in the context of second language acquisition. After a period of predominate focus on its quantitative aspects (e.g. how many times a new word must appear so that it is acquired), the attention has turned to the qualitative properties of the context in which the new word appears: „The quality of the context provides an answer to why gains in knowledge of meaning have varied from word to word (Horst et al. 1998; Saragi et al. 1978) and study to study (Horst et al 1998; Rott 1999; Saragi et al. 1978; Waring \& Takaki 2003)“ (Webb, 2008).

In the present study, we explore the incidental acquisition of German nouns in syntactically complex and simple contexts by adult native speakers.

\section{Experiments}

Sixty-eight native speakers of German participated in all experiments. In Experiment 1, 17 super-participants (each consisting of four participants that together formed a complete experimental list) read 20 short texts (ca. 93 words), each containing one to-be-learned word (TBLW, repeated three times in the text) that was represented by a pseudo-word taking the place of a real low-frequency word. The texts were constructed such that the meaning of the TBLW could be derived from its context.

ExLing 2012: Proceedings of 5th Tutorial and Research Workshop on Experimental Linguistics, 27-29 August 2012, Athens, Greece 
Each subject saw half of the texts in a syntactically complex version (long clauses, passive voice, infinitive and participle constructions) and the other half in a syntactically simple version (short main clauses). Each text was followed by 1-3 sentences that were read according to the self-paced reading method (moving window). One of the sentences contained the TBLW, which was combined either with a semantically plausible or implausible adjective, e.g.: kaputte Schocht (broken schocht (i.e. 'rake')) vs. leere Schocht (empty schocht (i.e. 'rake')).

In Experiment 2, the same participants performed a lexical decision task, in which the 20 TBLW appeared together with 20 pseudo-words and 20 real words.

In the semantic priming Experiment 3, the TBLWs were presented as primes in a semantic condition (the target was a word semantically related to the TBLW) and in a semantic + contextual condition (the target was semantically related and had also appeared three times together with the TBLW in the short texts of Experiment 1). Each related condition also had a respective counterpart with semantically unrelated primes and targets. In addition to the previous two conditions, there was also a control semantic condition with known primes and targets that did not appear in the previous experiments.

\section{Results}

The results of Experiment 1 show that participants read the syntactically complex texts significantly more slowly than the simple ones $(29,7 \mathrm{~s}$ vs. $28,3 \mathrm{~s})$. In addition, reading times during the self-paced reading part of the experiment were significantly slower $(\mathrm{p}<.01)$ in the implausible than in the plausible condition in the part of the text directly following the TBLW. This suggests that participants successfully derived the meanings of the TBLWs because they had problems to integrate them with the semantically implausible adjectives.

Moreover, a tendency towards an interaction between the factors plausibility and complexity at the position TBLW $+1(\mathrm{~F} 1(1,15)=6.9, \mathrm{p}<.05$; $\mathrm{F} 2(1,19)=2.2, \mathrm{p}=.15)$ suggests that subjects were less successful in deriving and/or representing the meaning of the TBLW in syntactically complex texts. 
Table 1. Results of Experiment 1; mean RTs in ms in all four conditions; effect of plausibility

\begin{tabular}{lllll}
\hline \hline & & \multicolumn{3}{c}{ Plausibility } \\
Position & & plausible & implausible & diff. \\
\hline TBLW & simple & 412,75 & 416,95 & 4,20 \\
& complex & 418,52 & 421,49 & 2,97 \\
\hline TBLW+1 & simple & 406,04 & 444,86 & 38,82 \\
& complex & 414,45 & 427,72 & 13,27 \\
\hline TBLW+2 & simple & 396,85 & 414,36 & 17,51 \\
& complex & 396,50 & 417,98 & 21,48 \\
\hline \hline
\end{tabular}

This finding is supported by the results of Experiment 2: Participants recognized the phonological form of the TBLWs significantly more often if the TBLW appeared in a syntactically simple context than if it appeared in a complex one. Analyses of RTs further revealed that the yes-responses to the TBLWs were slower than the yes-responses to the real words and even slower than the no-responses to the pseudowords. This indicates that participants successfully stored the phonological form of about $44 \%$ of the TBLWs, but access to them was still very demanding.

Table 2. Results of Experiment 2, mean RTs in ms and correct responses ("yes" for TBLWs)

\begin{tabular}{lccc}
\hline \hline & real words & pseudowords & TBLWs \\
\hline RT (in ms) & $617(99 \%)$ & $833(86 \%)$ & $941(44 \%)$ \\
\hline \hline
\end{tabular}

The results of Experiment 3 showed significant semantic priming effects in the control semantic condition. Subjects were significantly faster in recognizing familiar words in the semantically related than the semantically unrelated condition. However, no priming effects were observed when TBLWs appeared as primes, neither in the semantic, nor in the semantic + contextual condition. (For each subject only the RTs of the TBLWs that they identified in Experiment 2 as real words were analyzed).

\section{Conclusions}

The results of the self-paced reading Experiment 1 show that participants successfully derived the meaning of the TBLWs which appeared three times in short texts. The tendency towards larger and more immediate plausibility effects for TBLWs that appeared in syntactically simple contexts indicates that syntactic complexity interferes with the process of meaning derivation from context and/or with the process of creating the new representation. 
Moreover, the absence of the semantic priming effects for TBLWs in Experiment 3 suggests that the integration of the new TBLWs into the semantic network either had not taken place (yet), or that the connections were so weak that no effects could be measured.

The results of Experiment 2 show that participants established a formbased, graphemic representation for at least $44 \%$ of the TBLWs, suggesting that formal aspects were also memorized and retrievable independently from the context of acquisition. However, as indicated by the slow reaction times, access to these newly learned forms was very demanding. The larger number of recognized phonological forms that appeared in syntactically simple contexts indicates that syntactic complexity also had a negative effect on creating the phonological/graphemic representation of the TBLWs.

\section{Acknowledgements}

This research was supported by a DFG grant to Denisa Bordag: Project BO 3615/2-1 "Beiläufiger Erwerb von lexikalischem Wissen beim Lesen in Deutsch als Fremdsprache".

\section{References}

Horst, M., Cobb, T., and Meara, P. 1998. Beyond A Clockwork Orange: Acquiring second language vocabulary through reading. Reading in a Foreign Language, $11(2), 207-223$.

Laufer, B. 2001. Reading, word-focused activities and incidental vocabulary acquisition in a second language. Prospect 16 (3), 44-54.

Paribakht, T. S. and Wesche, M. 1996. Enhancing vocabulary acquisition through reading: A hierarchy of text-related exercise-types. The Canadian Modern Language Review, 52(2), 155-178.

Rott, S. 1999. The effect of exposure frequency on intermediate language learners incidental vocabulary acquisition through reading. Studies in Second Language Acquisition, 21(4), 589-619.

Saragi, T., Nation, I.S.P., and Meister, G.F. 1978. Vocabulary learning and reading. System, 6 (2), 72-78.

Waring, R. and Takaki, M. 2003. At what rate do learners learn and retain new vocabulary from, reading a graded reader? Reading in a Foreign Language, 15(2), $130-163$.

Webb, S. 2008. The effects of context on incidental vocabulary learning. Reading in a Foreign Language, 20(2), 232-245. 